1. Plemmons RM, Dooley DP, Longfield RN. Septic thrombophlebitis of the portal vein (pylephlebitis): Diagnosis and management in the modern era. Clin Infect Dis 1995; 21: 1114-20.

2. Singh P, Yadav N, Visvalingam V, Indaram A, Bank S. Pylephlebitis: Diagnosis and management. Am J Gastroenterol 2001; 96: 1312-3.

3. Saxena R, Adolph M, Ziegler JR, et al. Pylephlebitis: a case report and review of outcome in the antibiotic era. Am J Gastroenterol 1996; 91: 1251-6.

4. Baril N, Wren S, Radin R, Ralls P, Stain S. The role of anticoagulation in pylefphlebitis. Am J Surg 1996; 172: 449-53.

5. Ohtake H, Urayama H, Nozaki Z, Harada T, Kawasuji M, Wantanabe Y. Surgical drainage for idiopathic suppurative pylephlebitis. Ann Vasc Surg 1998; 12: 83-5.

6. Sillero JM, Calvet X, Musulen E, Diaz-Ruiz MJ, Tolosa C, Pardo A Corcuera A, Malet A, Font J. Idiopathic pylephlebitis and idiopathic sclerosing peritonitis in a man with protein $\mathrm{S}$ deficiency. J Clin Gastroenterol 2001; 32: 262-5.

7. Etienne M, Gueit I, Abboud P, Pons JL, Jacquot S, Caron F. Fusobacterium nucleatum hepatic abscess with pylephlebitis associated with idiopathic CD4+ T lymphocytopenia. Clin Infect Dis 2001; 32: 326-8.

8. Duffy FJ, Millan MT, Schoetz DJ, Larsen CR. Suppurative pylephlebitis and pylethrombosis: the role of anticoagulation. Am Surgeon 1995; 61: 1041-4.

9. Seeto RK, Rockey DC. Pyogenic liver abscess. Changes in etiology, management and outcome. Medicine 1996; 75: 99-106.

10. Condat B, Pessione F, Denninger MH, Hillaire S, Valla D. Recent portal and mesenteric venous thrombosis: increased recognition and frecuent recanalization on anticoagulant therapy. Hepatology 2000; 32: 466-70.

\section{Infarto mesentérico de etiología no oclusiva}

\section{Señor Director:}

La isquemia mesentérica no oclusiva es un tipo de isquemia mesentérica poco frecuente que presenta unas características diferenciales, y a menudo su diagnóstico se realiza por exclusión (1). Presentamos nuestra experiencia en esta patología.

Paciente de 82 años intervenido de carcinoma de laringe 4 días previos a su ingreso en UCI, con buena evolución en el postoperatorio inmediato. Presenta súbitamente en planta emisión de vómitos de considerable cuantía $(1.500 \mathrm{cc})$, así como crisis de arritmia completa por fibrilación auricular a 200 por minuto, con gran descenso en las cifras de tensión arterial $(50 / 30 \mathrm{mmHg})$, por lo que ingresa en la Unidad de Cuidados Intensivos, donde además de lo mencionado se aprecia dolor abdominal difuso de manera espontánea y también a la palpación. Se implanta sonda nasogástrica obteniendo un elevado débito por la misma (2.000 cc en las primeras 24 horas). Se instaura tratamiento con expansores del plasma (coloides) y posteriormente con drogas vasoactivas ante la refractariedad para la corrección de la tensión arterial. Así mismo se asocia amiodarona en perfusión continua para disminuir la frecuencia cardíaca. Ante desaturación importante es preciso iniciar ventilación mecánica. Se recibe analítica con valores incrementados de las enzimas hepáticas (AST 300 U/L; ALT $267 \mathrm{U} / \mathrm{L})$, urea $(1,30 \mathrm{~g} / \mathrm{l})$ y creatinina $(4 \mathrm{mg} / \mathrm{dl})$, encontrando también alteraciones en la coagulación (tiempo de protrombina 16 seg. actividad de protrombina $62,4 \%$, INR 1,49 , tiempo de tromboplastina parcial activada $36 \mathrm{seg}$, fibrinógeno $161 \mathrm{mg} / \mathrm{dl}$, plaquetas $55 \mathrm{mill} / \mathrm{mm}^{3}$ ). Se realiza una TAC abdominal de urgencia donde se aprecia una distensión generalizada de las asas intestinales, sin patología obstructiva subyacente, hallando unos infartos isquémicos múltiples en el parénquima hepático y esplénico
(Fig. 1). No existen alteraciones en la raíz de las ramas de la aorta. Como la situación persiste tras 24 horas de su ingreso, se realiza una laparotomía exploradora en la que no se objetiva sufrimiento de las asas intestinales ni anomalías en la vasculatura abdominal. El paciente evoluciona satisfactoriamente pudiendo retirar drogas vasoactivas y apoyo respiratorio tras una semana de evolución, con corrección de todas las alteraciones analíticas mencionadas.

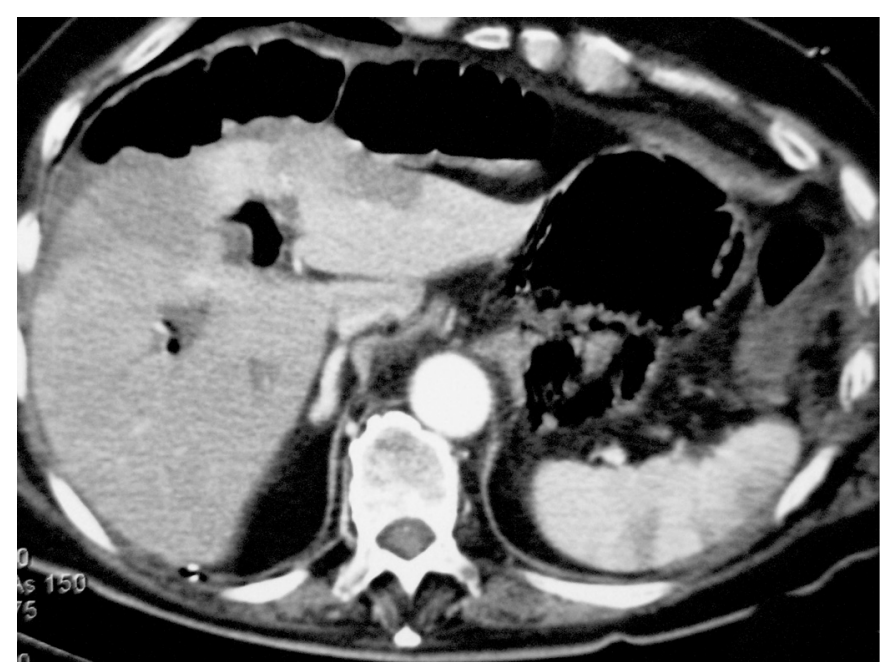

Fig. 1.TAC abdominal: distensión de asas intestinales. Infarto isquémico en parénquima hepático.

La isquemia mesentérica no oclusiva afecta a pacientes con edad superior a 50 años, constituye el $20-30 \%$ de todos los casos de isquemia mesentérica y posee una mortalidad del $50 \%$ que se incrementa con la edad, llegando a ser del $100 \%$ en los pacientes octogenarios $(1,2)$.

Habitualmente en su desarrollo concurren factores como insuficiencia cardiaca congestiva, insuficiencia aórtica, o fibrilación auricular, siendo característica la existencia de una intervención quirúrgica reciente (1). Puede existir, aunque no siempre, dolor abdominal como forma de presentación clínica, así como la presencia de íleo intestinal (ambos existentes en el caso presentado) (1,2).

$\mathrm{Su}$ mecanismo etiopatogénico reside en un fallo cardiaco agudo que origina una hipoperfusión intestinal a la que contribuyen significativamente la existencia de una fibrilación auricular o de una cirugía reciente, ambos presentes en nuestro caso. La fibrilación auricular en este tipo de isquemia, no juega un papel embolígeno, sino que es la responsable directa de una disminución en el flujo sanguíneo de la arteria mesentérica, lo que provoca una hipoperfusión de dicho territorio secundaria a la reducción de la función ventricular izquierda y por tanto de la fracción de eyección $(1,3)$. Por este motivo, es típica la aparición de isquemia en otros órganos abdominales, coexistiendo sincrónicamente infartos a nivel hepático, esplénico o renal (lo que explicaría los múltiples infartos hepáticos y esplénicos que se hallaron en el paciente) (1). La cirugía reciente es otro factor de riesgo para desarrollar este tipo de isquemia, probablemente por que comporta una pérdida de fluidos a menudo desapercibida, y un desarrollo de hipotensión arterial, ambos más frecuentes en pacientes ancianos (1). En ocasiones, existe también un vasoespasmo asociado de las arterias implicadas que se origina como mecanismo compensador, y que no se resuelve hasta que no se realiza la corrección de los factores precipitantes que han desencadenado la isquemia; dicho vasoespasmo puede también 
responder a la inyección intraarterial in situ de agentes vasodilatadores (2).

Métodos no invasivos como la TAC, RNM o ultrasonidos son capaces de valorar el estado de la aorta y la raíz de las arterias esplácnicas, aunque a pesar del desarrollo técnico de estos métodos la angiografía selectiva constituye el exámen de elección (2).

En este tipo de isquemia, a diferencia de la enfermedad oclusiva mesentérica no se encuentra indicado el tratamiento quirúrgico, salvo en los pacientes que presentan peritonitis $(1,2)$. Desafortunadamente ante la persistencia de una gran afectación del estado general y ausencia de datos objetivos que la justifiquen, un elevado porcentaje de pacientes son sometidos a una laparotomía exploradora sin encontrar hallazgos significativos, a lo sumo se ha descrito la existencia de infartos limitados a segmentos muy reducidos del tramo intestinal, añadiendo una nueva agresión quirúrgica para el paciente (1-3).

El manejo de esta patología requiere una aproximación multidisciplinar, con un soporte vital agresivo que permita la administración de una fluidoterapia enérgica para lograr el restablecimiento del flujo arterial y de la perfusión mesentérica, y en ocasiones una realización de angiografía selectiva, e incluso la administración local de agentes vasodilatadores. La laparotomía exploradora queda relegada a los pacientes que presentan peritonitis o infarto intestinal $(1,4)$.

\section{B. Obón Azuara, I. Gutiérrez Cía, B. Villanueva Anadón, N. Sánchez Donoso, R. Montoiro Allué}

Servicio de Medicina Intensiva. Hospital Clínico Universitario. Zaragoza

1. Acosta S, Ogren M, Sternby NH, Bergqvist D, Bjorck M. Fatal nonocclusive mesenteric ischaemia: population-based incidence and risk factors. J Intern Med 2006; 259: 305-13.

2. Trompeter M, Brazda T, Remy CT, Vestring T, Reimer P. Non-occlusive mesenteric ischemia: etiology, diagnosis, and interventional therapy. Eur Radiol 2002; 12: 1179-87.

3. John AS, Tuerff SD, Kerstein MD. Nonocclusive mesenteric infarction in hemodialysis patients. J Am Coll Surg 2000; 190: 84-8.

4. Kolkman J, Mensink P. Nonocclusive mesenteric ischaemia: a common disorder in gastroenterology and intensive care. Best Pract Res Clin Gastroenterol 2003; 17: 457-73.

\section{Síndrome de Guillain-Barre como forma de presentación de carcinoma escamoso de pulmón}

\section{Sr. Director:}

En la literatura está descrita la asociación entre carcinomas y diversas formas de neuropatía periférica de origen paraneoplásico, que en muchos casos precede al diagnóstico del tumor. Dentro de las diversas formas de afectación neurológica el síndrome de Guillain-Barré (SGB) también ha sido reportado, aunque con menor frecuencia. Presentamos un caso de SGB que se presentó de modo coincidente con el diagnóstico de carcinoma escamoso de pulmón.

Paciente de 83 años de edad con antecedentes de tabaquismo activo (1 paquete/día, desde hacia 20 años) y pólipos laríngeos detectados hacía 10 años. Ingresa por cuadro de tres días de evolución consistente en parestesisas, dolor y debilidad en extremidades inferiores de carácter ascendente, con incapacidad para la deambulación. En la exploración física presentaba unas constantes normales y destacaba la presencia de crepitantes en la base pulmonar izquierda a la auscultación así cómo una tetraparesia flácida de predominio proximal y arreflexia difusa, con sensibilidad conservada, en la exploración neurológica. El hemograma y la bioquímica sanguínea eran normales, en la TAC craneal presentaba signos difusos de atrofia córtico-subcortical, en la radiografía de tórax se apreciaba una condensación basal izquierda. Se practicó TAC torácico (Fig. 1) que mostró una masa de $5 \mathrm{x} 4 \mathrm{~cm}$. en lóbulo inferior izquierdo sugestiva de tumoración que en la broncoscopia producía signos de compresión extrínseca e infiltración submucosa en dicho lóbulo. La citología del BAS fue positiva para células malignas compatibles con carcinoma escamoso de pulmón. El electromiograma fue informado como polirradiculoneuritis de mayor afectación en EEII. En el líquido cefalorraquídeo presentaba: proteinas $96 \mathrm{mg} / \mathrm{dl}$, leucocitos $3 / \mathrm{mm}^{3}$, glucosa $80 \mathrm{mg} / \mathrm{dl}$. Se inició tratamiento con gammaglobulinas endovenosas objetivándose una importante mejoría clínica neurológica a corto plazo. Respecto al tumor la familia decidió no iniciar terapeuticas agresivas por lo que finalmente se derivó al paciente para tratamiento paliativo domiciliario.
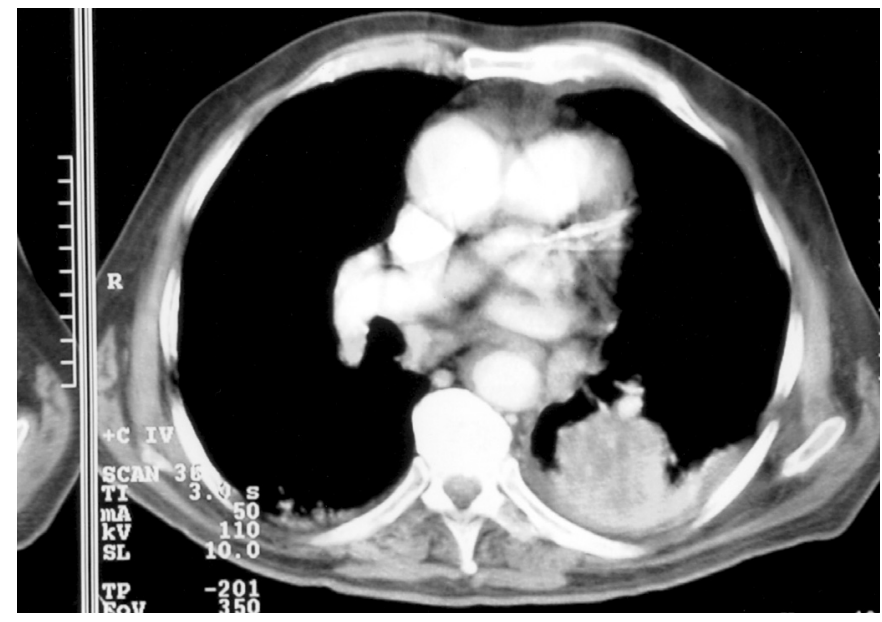

Fig. 1. TAC torácico mostrando una masa en lóbulo inferior izquierdo sugestiva de tumoración.

Cualquier parte del sistema nervioso puede verse afectada por un síndrome paraneoplásico, aunque son entidades raras que se estima afectan a sólo el $1 \%$ de pacientes con cáncer (1). En algunos casos la presencia de determinados anticuerpos anti- onconeurales son de gran ayuda para establecer la asociación, como sucede con el Anti-HU y el tumor pulmonar de células pequeñas. No obstante, existen verdaderas neuropatías paraneoplásicas sin un anticuerpo relacionado (2). Respecto al SGB, se han publicado casos cómo el nuestro asociados a cáncer, algunos en nuestro país (3), aunque la existencia de un SGB paraneoplásico no se ha confirmado. En un estudio poblacional italiano reciente se comparó en una cohorte de 435 pacientes diagnosticados de SGB la frecuencia de casos de cáncer detectados respecto a los esperados en la cohorte de acuerdo a los datos del registro de la población general (4). Se detectaron 9 casos de cáncer (tres de ellos eran tumores pulmonares no de células pequeñas), siendo la cifra esperada de 3,8, lo que supone un riesgo relativo de 2,37 (IC 95\% $1,09-4,05)(\mathrm{p}<0,01)$. Los autores concluyen que la asociación no 